University of Nebraska - Lincoln

DigitalCommons@University of Nebraska - Lincoln

Norman R. Simon Papers

Research Papers in Physics and Astronomy

1988

\title{
Photometry of variable stars with periods near 1 day
}

Charles G. Loomis

University of Nebraska - Lincoln

Edward G. Schmidt

University of Nebraska at Lincoln, eschmidt1@unl.edu

Norman R. Simon

University of Nebraska - Lincoln, nsimon@unl.edu

Follow this and additional works at: https://digitalcommons.unl.edu/physicssimon

Loomis, Charles G.; Schmidt, Edward G.; and Simon, Norman R., "Photometry of variable stars with periods near 1 day" (1988). Norman R. Simon Papers. 49.

https://digitalcommons.unl.edu/physicssimon/49

This Article is brought to you for free and open access by the Research Papers in Physics and Astronomy at DigitalCommons@University of Nebraska - Lincoln. It has been accepted for inclusion in Norman R. Simon Papers by an authorized administrator of DigitalCommons@University of Nebraska - Lincoln. 


\title{
Photometry of variable stars with periods near 1 day
}

\author{
Charles Loomis, Edward G. Schmidt and \\ Norman R. Simon Behlen Observatory, Department of Physics and \\ Astronomy, University of Nebraska, Lincoln, NE 68588, USA
}

Accepted 1988 May 6. Received 1988 April 29; in original form 1988 January 25

\begin{abstract}
Summary. We have obtained accurate, well-sampled light curves for seven stars which were candidates for short period type II Cepheids. One of the stars, CM UMa, was found to have an erroneous period. The correct period is 0.589 day and the star is an RRab star. The Fourier decomposition coefficients for the light curve of KP Cyg (period $=0.86$ day) combined with its high metallicity are consistent with its being either a type II Cepheid or an RR Lyrae star. The result for V486 Her (period $=0.8$ day) is inconclusive; it is probably an RRab star. The Fourier coefficients showed that CEHer (period=1.21 day) and XX Vir (period=1.35 day) are type II Cepheids as suspected. Both stars show excess scatter and rapid variations during declining light which we suggest deserve further attention. BB Gem has Fourier coefficients which are appropriate to a short period classical Cepheid, making it the shortest period fundamental pulsator of that class known in the Galaxy.
\end{abstract}

\section{Introduction}

A period of 1 day is commonly assumed as the boundary between the RRab stars and the type II Cepheids. The latter stars in the short-period range are a heterogeneous group with metallicities ranging from very low to at least solar (Harris 1981), kinematics appropriate to a range of populations (Harris \& Wallerstein 1984) and a variety of light curve shapes. Diethelm (1983) sorted the light curves into several categories and subsequently (Diethelm 1986) used photometric abundances to show that at least one of the groups, his RRd stars, was physically distinct.

Petersen \& Diethelm (1986) carried out Fourier decomposition of the light curves of 35 type II Cepheids and concluded that their pulsational properties were similar to those of the classical Cepheids. However, Simon (1986) rediscussed the Fourier coefficients in connection with pulsation models and reached somewhat different conclusions. In particular, he suggested that there 
may be mass differences between some of Diethelm's groups. We are led to conclude that among pulsating stars with periods near 1 day there are several different types of object.

Although these studies have provided new insights, it is clear that more observations are needed. An examination of the light curves (see Diethelm 1983 for plots) shows that the data, drawn from a number of sources, often contain excessive scatter, exceeding 0.1 mag in some cases. For some stars there are gaps in the phase coverage which can introduce difficulties for Fourier decomposition. Furthermore, the sample of usable light curves is rather small. Peterson \& Diethelm considered only five of the 35 light curves they decomposed to be of high quality.

To improve this situation we have started a program of photometric observations of variable stars with periods from about 0.75 day to 1 week. While the long-period limit is consistent with the usual division between BLHer and WVirstars, we have extended the sample to somewhat shorter periods since, as Wallerstein \& Cox (1984) pointed out, terminating the type II Cepheid regime at 1 day is an essentially arbitrary choice.

This paper presents results for the seven stars listed in Table 1. The first column gives the star name while columns two and three give the period and type of variability from the General Catalogue of Variable Stars (4th edn). As discussed below we have determined new epochs of maximum for all except one of them. These are given in column four while column five indicates the type of variability inferred from our data.

Table 1. The variable stars.

\begin{tabular}{|c|c|c|c|c|c|}
\hline & Star & Period & $\begin{array}{l}\text { Type } \\
\text { (GCVS) }\end{array}$ & $\begin{array}{l}\text { Epoch of } \\
\text { Maximum * }\end{array}$ & $\begin{array}{c}\text { Type } \\
\text { (revised) }\end{array}$ \\
\hline$C M$ & UMa & 0.589 & Cep: & $\begin{array}{l}6057.92 \\
6442.03 \\
7196.69\end{array}$ & $\mathrm{RRab}$ \\
\hline FK & Vul & 0.4340529 & RRab & .88 & RRab \\
\hline V48 & 36 Her & 0.8059317 & RRab & 6624.83 & RRab? \\
\hline $\mathrm{KP}$ & Cyg & 0.855936 & RRab & 6599.62 & Type II Cepheid or RR Lyrae \\
\hline$C E$ & Her & 1.2094357 & CWB & 6583.77 & Type II Cepheid, S-XX \\
\hline$x x$ & Vir & 1.3482051 & CWB & 6203.67 & Type II Cepheid, S-XX \\
\hline $\mathrm{BB}$ & Gem & 2.308207 & DCep & 6056.78 & Classical Cepheid \\
\hline \multicolumn{6}{|c|}{$\begin{array}{l}\text { Determined from present data, Heliocentric Julian } \\
\text { Date - 2,440,000. For CM UMa, the period determined } \\
\text { with the present data was not accurate enough to carry } \\
\text { the phasing from one season to the next. For that } \\
\text { reason, we give a date of maximum for each year it } \\
\text { was observed. }\end{array}$} \\
\hline
\end{tabular}

\section{The observations}

We made most of the observations with the automatic filter photometer (Taylor 1980) on the Behlen Observatory 0.76-m telescope. An RCA 31034C photomultiplier was used with broadband $V$ - and $R$-filters matched to the Cousins system. Both the telescope and the photometer were under computer control. This allowed rapid switching between the star and sky apertures and between the variable and its comparison star. It was thus possible to obtain accurate differential magnitudes under somewhat adverse conditions.

Measurements of the variable through both the $V$ - and $R$-filter were alternated with observations of the comparison star. A sequence consisted of at least four observations of the variable and continued until the internal errors of the differentials were judged to be satisfactory. The only exception to this practice occurred when rapid changes in the variable were noted. In that case, the sequence was continued as long as possible.

On photometric quality nights we observed standard stars from Landolt's (1983) list and 
determined transformations to the system of Cousins. The nights were grouped into intervals no longer than a month and mean colour terms calculated. These were then used for all the nights in the interval. When a night was regarded as photometric, zero points were also derived and the magnitudes were reduced to the standard system. For nights which were not of photometric quality, only differential magnitudes were derived but they are still on the standard system in the sense of having been corrected for colour terms. It was found that the mean colour terms for our filters and photocell changed by less than 0.02 mag over periods of months. Since this is smaller than the error in determining nightly transformations, the use of mean coefficients is justified and will reduce the internal scatter in the final results.

Once the observations had been placed on the standard system for the photometric nights or corrected for colour terms on the non-photometric nights, differential magnitudes were formed for the variables relative to the comparison stars. The observational sequence was generally

Table 2. Photometric data for CMUMa (period: 0.589, epochs of maximum: 2446057.92 and 2446442.03 ).

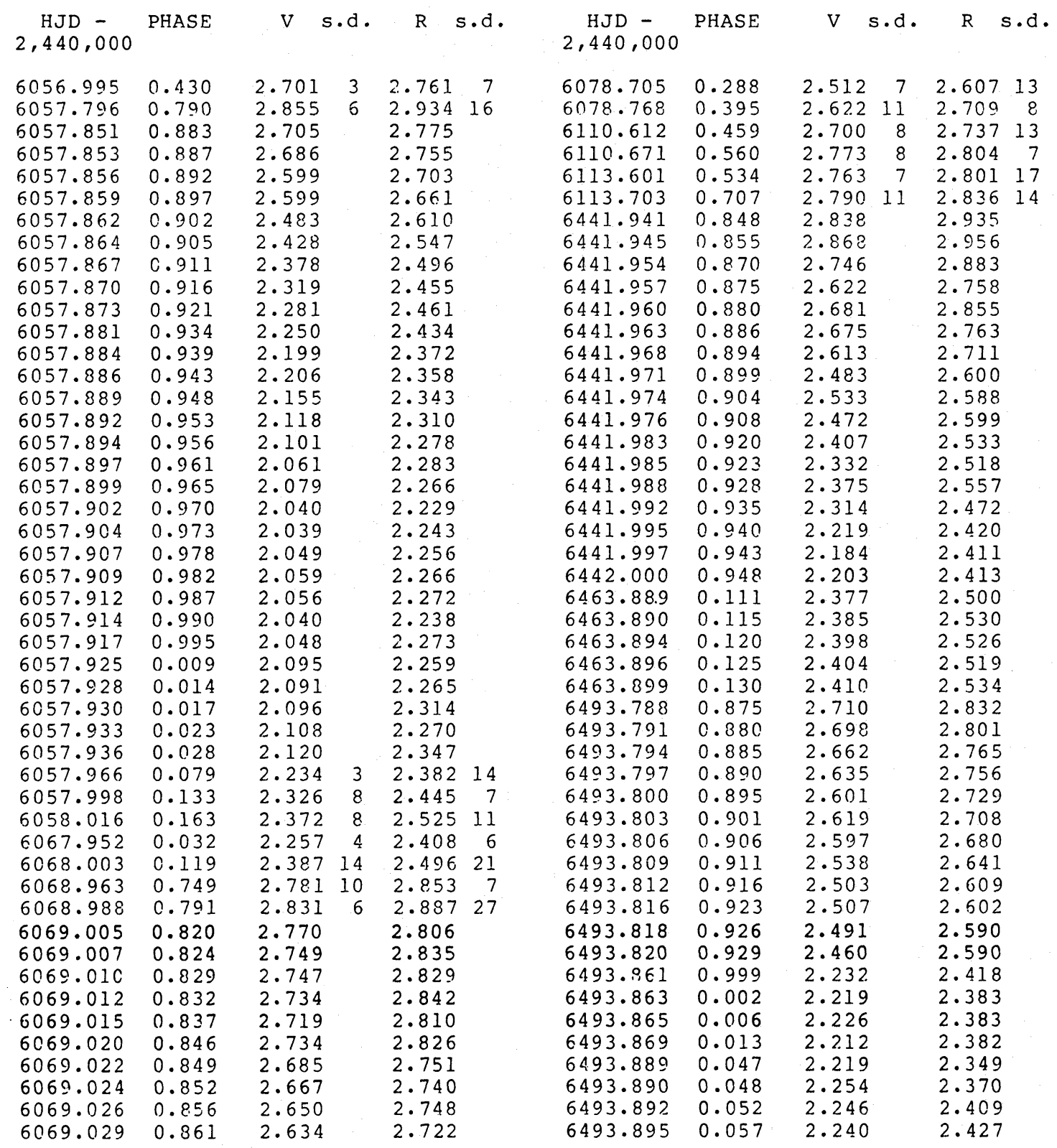


started and terminated with an observation of the variable, so usual practice was to form a differential magnitude for the mean of pairs of variable measurements and the intervening comparison star magnitude. The differential magnitudes were then averaged for the sequence and the root mean square of the deviations from the mean calculated. This rms value was then increased by the square root of two since there are only $n / 2$ statistically independent data points in $n$ differential magnitudes.

The differential magnitudes of the seven variable stars and their internal standard deviations are listed in Tables 2-8 and are plotted in Figs 1-7. The phases were calculated from the GCVS periods (column 2 of Table 1) and the epochs of maximum determined from our data (column 4).

In instances when the variable changed significantly between successive observations, differential magnitudes from single observations are given in Tables 2-8. Such cases can be recognized because they lack an entry in the standard deviation column. Observations of this type were only retained when they defined parts of the light curve not otherwise available and when the sky conditions were judged to be stable from the comparison star observations.

In order to verify the constancy of the comparison stars, a check star was selected for each (with

Table 3. Photometric data for FK Vul (period: 0.4340529, epoch of maximum: 2446593.88 ).

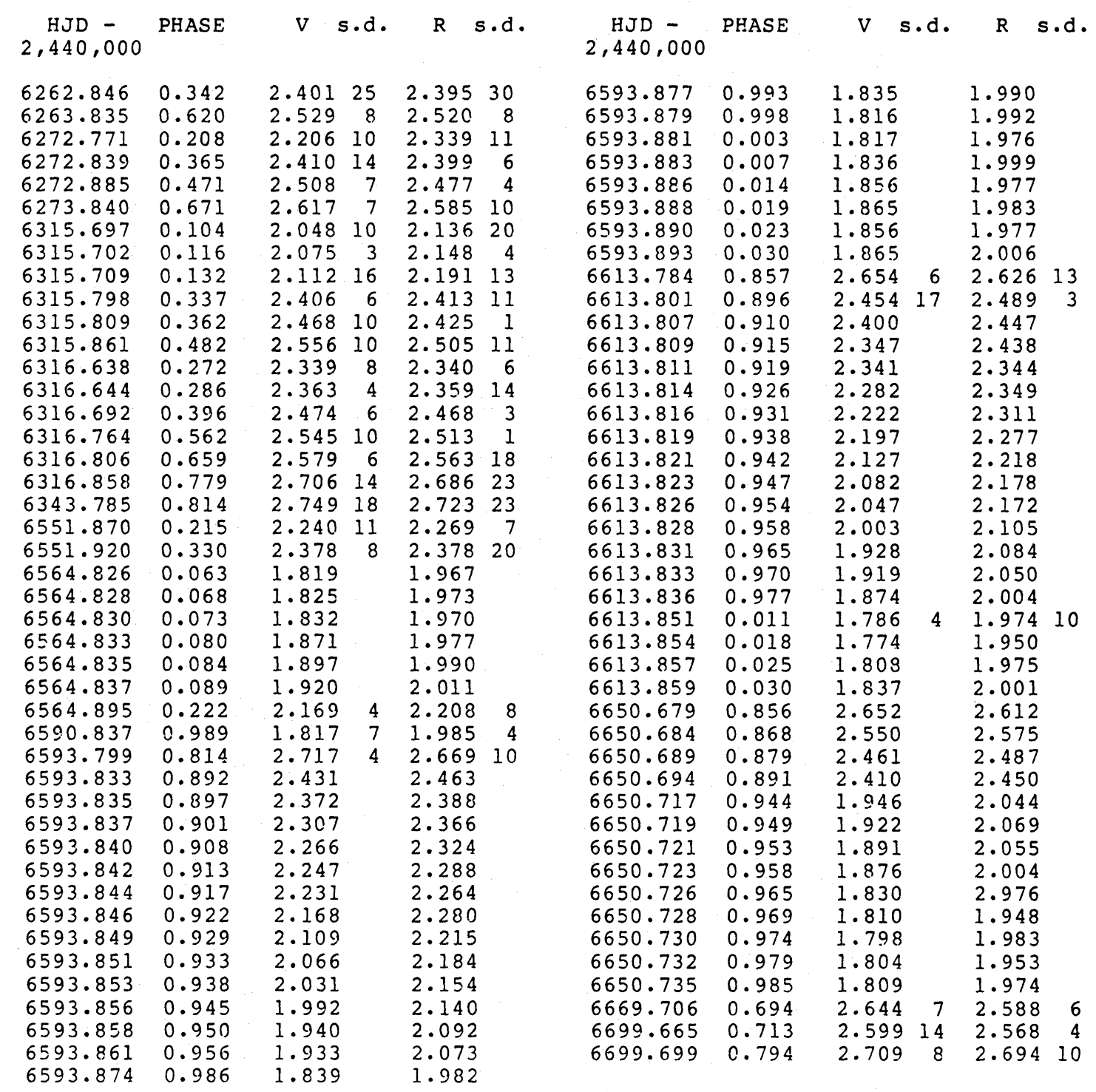


Table 4. Photometric data for V486 Her (period: 0.8059317, epoch of maximum: 2446624.83 ).

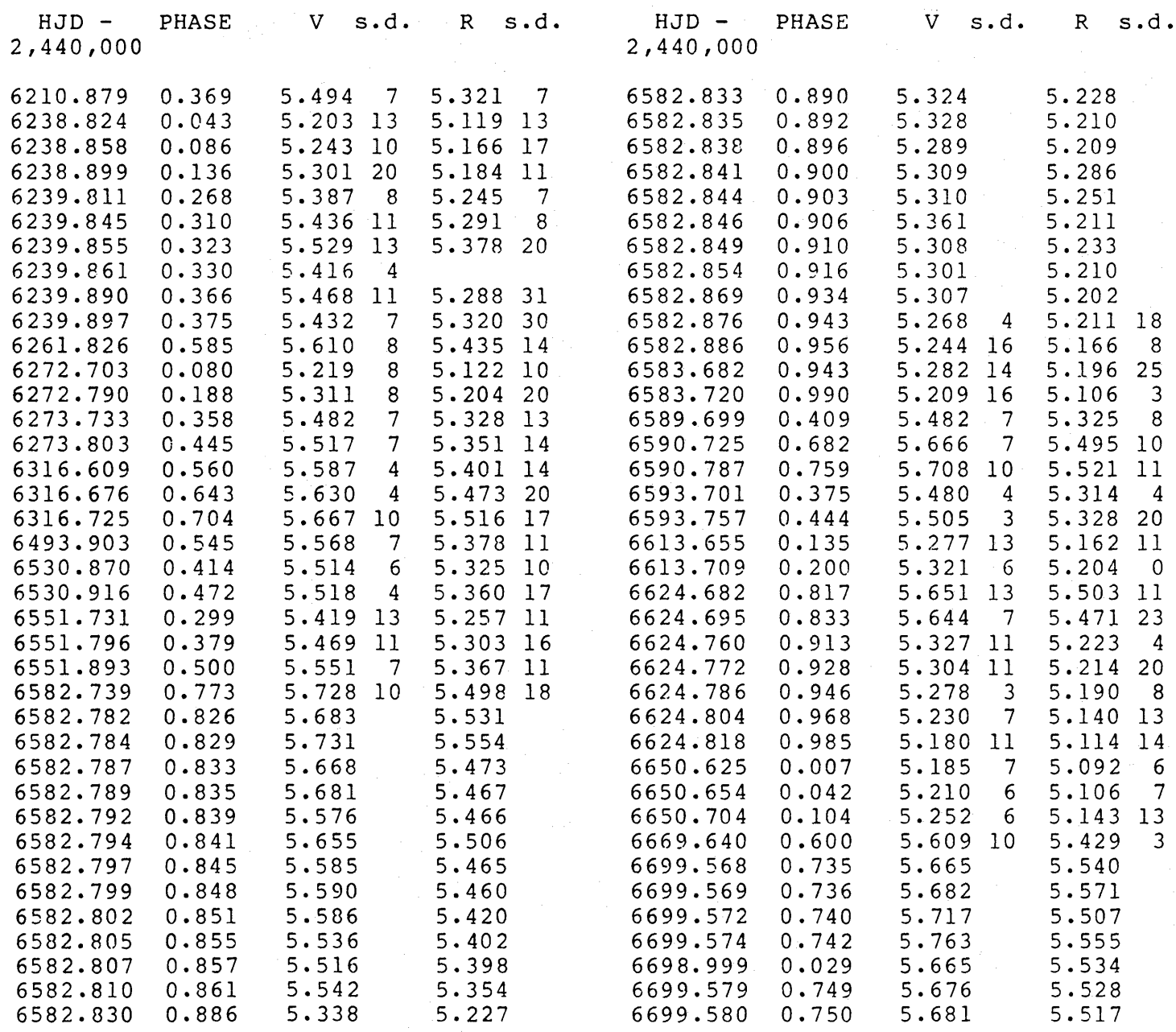

Table 5. Photometric data for KP Cyg (period: 0.855936, epoch of maximum: 2446 699.62).

\begin{tabular}{|c|c|c|c|c|c|c|c|c|c|c|}
\hline $\begin{array}{c}\text { HJD }- \\
2,440,000\end{array}$ & PHASE & V & s.d. & $\mathrm{R}$ & s.d. & $\begin{array}{c}\text { HJD } \\
2,440,000\end{array}$ & PHASE & V & s.d. & $R$ \\
\hline $\begin{array}{l}6272.736 \\
6272.814 \\
6272.865 \\
6272.902 \\
6273.872 \\
6302.744 \\
6302.756 \\
6316.622 \\
6316.706 \\
6316.752 \\
6316.832 \\
6343.688 \\
6343.752 \\
6551.841 \\
6590.819 \\
6590.894 \\
6590.896 \\
6590.898 \\
6590.900 \\
6590.903 \\
6596.814 \\
6699.589 \\
6699.590\end{array}$ & $\begin{array}{l}0.266 \\
0.357 \\
0.417 \\
0.460 \\
0.594 \\
0.325 \\
0.339 \\
0.539 \\
0.637 \\
0.690 \\
0.784 \\
0.160 \\
0.235 \\
0.349 \\
0.887 \\
0.975 \\
0.977 \\
0.979 \\
0.982 \\
0.985 \\
0.890 \\
0.964 \\
0.965\end{array}$ & $\begin{array}{l}3.350 \\
3.472 \\
3.541 \\
3.592 \\
3.791 \\
3.420 \\
3.457 \\
3.701 \\
3.841 \\
3.890 \\
3.948 \\
3.204 \\
3.314 \\
3.470 \\
3.757 \\
3.081 \\
3.068 \\
3.042 \\
3.045 \\
3.034 \\
3.697 \\
3.182 \\
3.180\end{array}$ & $\begin{array}{r}17 \\
8 \\
3 \\
7 \\
8 \\
10 \\
8 \\
6 \\
6 \\
7 \\
18 \\
16 \\
7 \\
8 \\
6 \\
\end{array}$ & $\begin{array}{l}3.438 \\
3.519 \\
3.569 \\
3.623 \\
3.769 \\
3.484 \\
3.513 \\
3.703 \\
3.823 \\
3.873 \\
3.921 \\
3.335 \\
3.412 \\
3.502 \\
3.784 \\
3.245 \\
3.245 \\
3.213 \\
2.205 \\
3.743 \\
3.343 \\
3.335\end{array}$ & $\begin{array}{r}16 \\
6 \\
4 \\
8 \\
7 \\
10 \\
8 \\
20 \\
16 \\
20 \\
8 \\
6 \\
10 \\
8 \\
13 \\
\\
\end{array}$ & $\begin{array}{l}6699.593 \\
6699.595 \\
6699.597 \\
6699.599 \\
6699.602 \\
6699.604 \\
6699.606 \\
6699.609 \\
6699.612 \\
6699.614 \\
6699.616 \\
6699.618 \\
6699.621 \\
6699.622 \\
6710.698 \\
6710.700 \\
6710.702 \\
6710.704 \\
6710.707 \\
6710.709 \\
6710.712 \\
6710.715 \\
6710.718\end{array}$ & $\begin{array}{l}0.969 \\
0.971 \\
0.973 \\
0.976 \\
0.979 \\
0.982 \\
0.984 \\
0.987 \\
0.991 \\
0.993 \\
0.996 \\
0.998 \\
0.001 \\
0.003 \\
0.942 \\
0.945 \\
0.947 \\
0.949 \\
0.953 \\
0.955 \\
0.959 \\
0.962 \\
0.966\end{array}$ & $\begin{array}{l}3.137 \\
3.109 \\
3.115 \\
3.105 \\
3.072 \\
3.031 \\
3.050 \\
3.027 \\
3.004 \\
3.018 \\
3.001 \\
2.982 \\
3.010 \\
3.007 \\
3.363 \\
3.350 \\
3.326 \\
3.323 \\
3.307 \\
3.275 \\
3.231 \\
3.174 \\
3.161\end{array}$ & & $\begin{array}{l}3.295 \\
3.310 \\
3.257 \\
3.269 \\
3.256 \\
3.243 \\
3.213 \\
3.217 \\
3.201 \\
3.214 \\
3.173 \\
3.169 \\
3.196 \\
3.194 \\
3.496 \\
3.494 \\
3.458 \\
3.436 \\
3.407 \\
3.393 \\
3.391 \\
3.345 \\
3.278\end{array}$ \\
\hline
\end{tabular}


Table 6. Photometric data for CE Her (period: 1.2094357, epoch of maximum: 2446583.77 ).

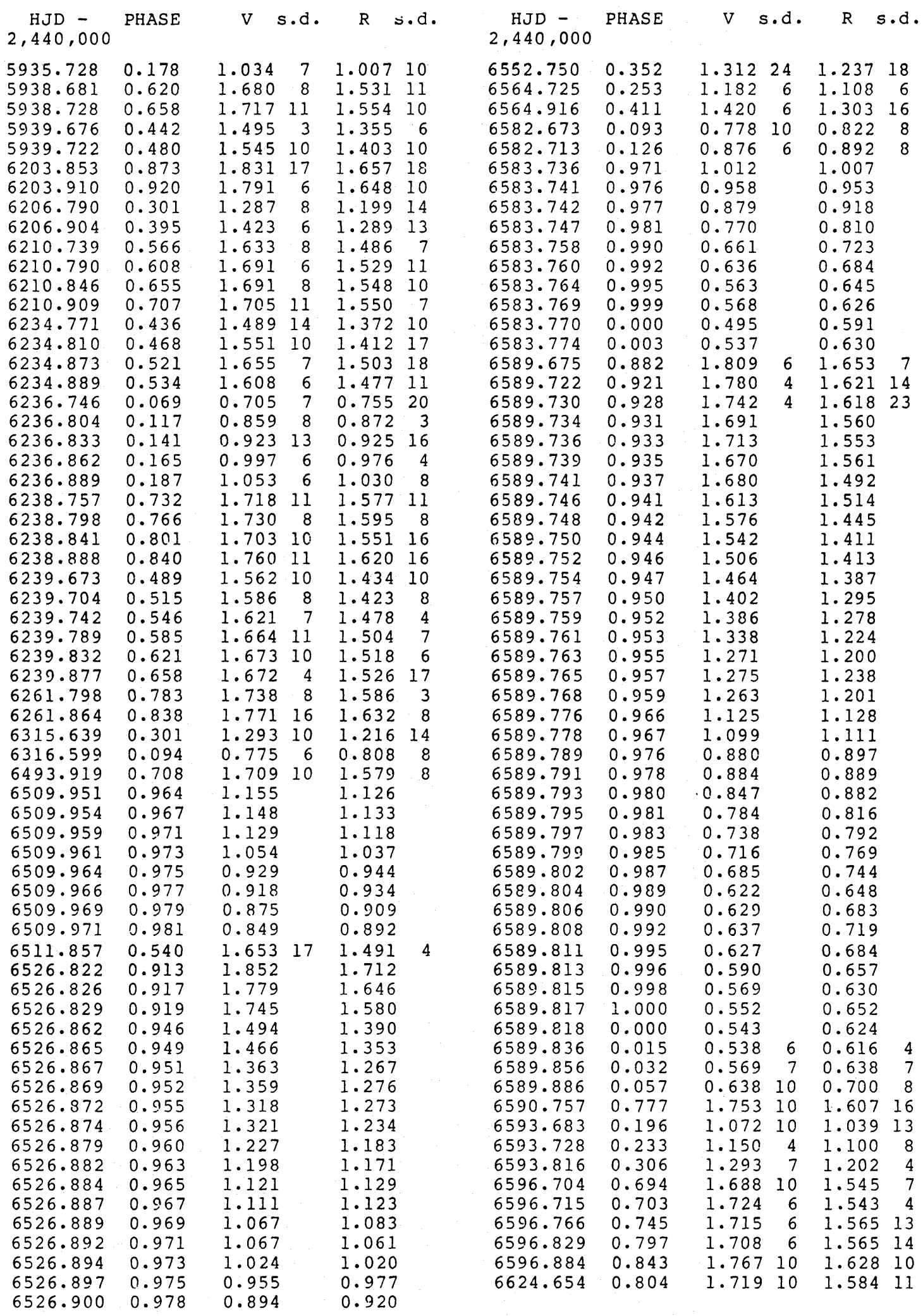

Figure 1. Light curves for CM UMa. The two upper panels present the data from the photoelectric photometry while the lower panel contains the data from the CCD photometry. All are phased with a period of 0.589 and the epoch from Table 1 which is appropriate to each observation. Here and in subsequent figures open circles denote points based on a single differential or points with standard errors larger than $0.01 \mathrm{mag}$. When a number of open circles are closely crowded, they are plotted as a single larger circle. 
Photometry of variable stars

Table 7. Photometric data for XX Vir (period: 1.3482051, epoch of maximum: 2446 203.67).

\begin{tabular}{|c|c|c|c|c|c|c|c|c|c|c|c|}
\hline $\begin{array}{r}\text { HJD }- \\
2,440,000\end{array}$ & PHASE & V & s.d. & $\mathrm{R}$ & s.d. & $\begin{array}{c}\text { HJD - } \\
2,440,000\end{array}$ & PHASE & V & s.d. & $\mathrm{R}$ & s.d. \\
\hline $\begin{array}{l}6141.872 \\
6171.749\end{array}$ & $\begin{array}{l}0.162 \\
0.323\end{array}$ & $\begin{array}{l}2.164 \\
2.396\end{array}$ & $\begin{array}{r}15 \\
8\end{array}$ & $\begin{array}{l}2.105 \\
2.286\end{array}$ & $\begin{array}{r}0 \\
10\end{array}$ & $\begin{array}{l}6552.791 \\
6552.822\end{array}$ & $\begin{array}{l}0.952 \\
0.975\end{array}$ & $\begin{array}{l}1.975 \\
1.749\end{array}$ & 10 & $\begin{array}{l}1.972 \\
1.785\end{array}$ & 10 \\
\hline 6171.806 & 0.365 & 2.463 & 10 & 2.335 & 14 & 6556.659 & 0.821 & 2.874 & 14 & 2.680 & 10 \\
\hline 6203.673 & 0.002 & 1.729 & 4 & 1.781 & 10 & 6556.668 & 0.828 & 2.848 & 11 & 2.674 & 17 \\
\hline 6203.724 & 0.040 & 1.846 & 8 & 1.864 & 10 & 6556.698 & 0.850 & 2.883 & 11 & 2.708 & 11 \\
\hline .768 & 0.073 & 1.945 & 3 & 1.944 & 6 & 6.745 & 0.885 & 2.840 & 8 & 2.680 & 13 \\
\hline & 0.111 & 2.035 & 10 & 2.012 & 8 & 6556.754 & 0.892 & 2.812 & & 2.685 & \\
\hline 6210.658 & 0.183 & 2.184 & 6 & 2.102 & 11 & 6556.778 & 0.909 & 2.595 & & 2.490 & \\
\hline 6210.711 & 0.222 & 2.255 & 10 & 2.172 & 4 & 5.780 & 0.911 & 2.571 & & 2.422 & \\
\hline 6210.774 & 0.269 & 2.342 & 6 & 2.233 & 13 & .782 & 0.912 & 2.555 & & 2.437 & \\
\hline 0.812 & 0.297 & 2.362 & 4 & 2.260 & 7 & .785 & 0.915 & 2.437 & & 2.321 & \\
\hline 6171.886 & 0.425 & 2.498 & 4 & 2.336 & 28 & 6.787 & 0.91 & 2.380 & & 2.275 & \\
\hline 6236.720 & 0.514 & 2.650 & 20 & 2.487 & 7 & 6.790 & 0.918 & 2.428 & & 2.329 & \\
\hline 85 & 0. & 2.734 & 6 & 2.578 & 16 & .792 & 0.9 & 2.367 & & 2.234 & \\
\hline 9.719 & 0.739 & 2.749 & 8 & 2.607 & 11 & 6.795 & 0.922 & 2.382 & & 2.266 & \\
\hline .837 & 0.224 & 2.262 & 10 & 2.190 & 10 & .798 & 0.924 & 2.389 & & 2.314 & \\
\hline .876 & 0.253 & 2.324 & 3 & 2.210 & 6 & .800 & 0 . & 2.365 & & 2.293 & \\
\hline 3.940 & 0.301 & 2.384 & 8 & 2.275 & 3 & .803 & 0 . & 2.368 & & 2.266 & \\
\hline 5493.961 & 0.316 & 2.410 & 3 & 2.296 & 8 & .805 & 0.929 & 2.337 & & 2.255 & \\
\hline 3.990 & 0.338 & 2.442 & 8 & 2.315 & 7 & .824 & 0.944 & 2.183 & & 2.107 & \\
\hline .825 & 0.083 & 1.993 & 10 & 1.985 & 8 & .826 & 0 . & 2.117 & & 2.093 & \\
\hline 6509.865 & 0.113 & 2.045 & 17 & 2.019 & 21 & .828 & 0.9 & 2.094 & & 2.055 & \\
\hline 9.922 & 0.155 & 2.168 & 11 & 2.122 & 14 & .831 & 0.9 & 2.054 & & 2.059 & \\
\hline 1.796 & 0.545 & 2.644 & 7 & 2.479 & 14 & .833 & 0.950 & 2.030 & & 2.058 & \\
\hline .827 & 0.568 & 2.676 & 7 & 2.519 & 8 & 35 & 0.952 & 2.048 & & 2.038 & \\
\hline .785 & 0.630 & 2.680 & 11 & 2.524 & 13 & .838 & 0.954 & 1.941 & & 1.994 & \\
\hline .935 & 0.741 & 2.733 & 10 & 2.586 & 8 & 1.653 & 0.750 & 2.776 & 7 & 2.604 & 11 \\
\hline .703 & 0.019 & 1.803 & 4 & 1.833 & 13 & .676 & 0.767 & 2.771 & 10 & 2.603 & 3 \\
\hline 6552.716 & 0.897 & 2.788 & 7 & 2.626 & 10 & .753 & 0.8 & 2.876 & 8 & 2.704 & \\
\hline 2.768 & 0.935 & 2.311 & & 2.249 & & 86 & 0.850 & 2.920 & 7 & 2.719 & \\
\hline 6552.772 & 0.938 & 2.258 & & 2.207 & & .802 & 0.861 & 2.884 & 4 & 2.710 & 0 \\
\hline 74 & 0.940 & 2.210 & & 2.186 & & 2.645 & 0.096 & 1.991 & 11 & 1.972 & 2 \\
\hline .776 & 0.9 & 2.198 & & 2.149 & & 0.675 & 0.052 & 1.891 & 7 & 1.888 & $8 \quad 23$ \\
\hline .779 & 0.943 & 2.155 & & 2.121 & & 4.701 & 0.038 & 1.841 & 4 & 1.884 & $4 \quad 10$ \\
\hline & & 2.153 & & 2.119 & & 6.676 & 0.503 & 2.626 & 10 & 2.455 & 5 \\
\hline & & & & 2.090 & & & & 2.671 & 6 & & \\
\hline & & & & 2.060 & & & & 2.060 & 11 & 2.024 & \\
\hline .78 & & & & 1.950 & & 4.665 & 0.264 & 43 & 6 & 2.231 & \\
\hline
\end{tabular}

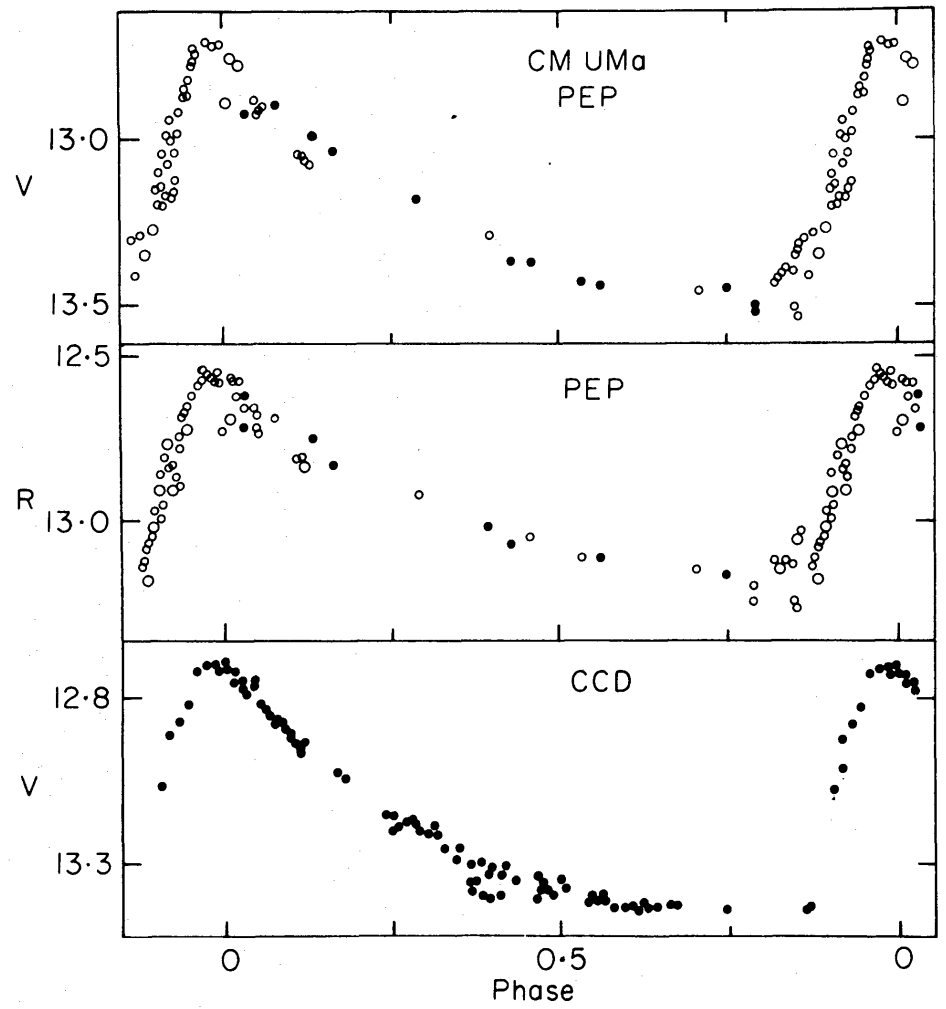

Figure 1. 
Table 8. Photometric data for BB Gem (period: 2.308207, epoch of maximum: 2446056.78 ).

\begin{tabular}{|c|c|c|c|c|c|c|c|c|c|c|c|}
\hline $\begin{array}{l}\text { HJD } \\
2,440,000\end{array}$ & PHASE & V & s.d. & $\mathrm{R}$ & s.d. & $\begin{array}{r}\text { HJD } \\
2,440,000\end{array}$ & PHASE & V & $5 \cdot d$. & $\mathrm{R}$ & . d. \\
\hline 6056.795 & 0.006 & 1.173 & 1 & 1.316 & 4 & 6439.776 & 0.928 & 1.776 & & 1.809 & \\
\hline 6056.879 & 0.043 & 1.204 & 6 & 1.331 & 4 & 6439.780 & 0.929 & 1.719 & & 1.740 & \\
\hline 6056.937 & 0.068 & 1.261 & 6 & 1.385 & 3 & 6439.782 & 0.931 & 1.705 & & 1.721 & \\
\hline 6057.662 & 0.382 & 1.903 & 3 & 1.862 & 4 & 6439.785 & 0.932 & 1.718 & & 1.739 & \\
\hline 6057.758 & 0.424 & 1.950 & 6 & 1.911 & 4 & 6439.789 & 0.933 & 1.734 & & 1.775 & \\
\hline 6057 & 0.455 & 1.975 & 7 & 1.929 & 7 & 6439.791 & 0.935 & 1.681 & & 1.720 & \\
\hline 6057.949 & 0.506 & 2.036 & 1 & 1.970 & 6 & 6439.797 & 0.937 & 1.615 & & 1.679 & \\
\hline 6067.923 & 0.327 & 2.168 & 6 & 2.099 & 6 & 6439.801 & 0.939 & 1.595 & & 1.660 & \\
\hline 6077.633 & 0.034 & 1.202 & 13 & ]. 334 & 13 & 6439.806 & 0.941 & 1.562 & & 1.643 & \\
\hline 6078.625 & 0.464 & 1.990 & 8 & 1.931 & 7 & 6439.811 & 0.943 & 1.544 & & 1.607 & \\
\hline 6078.682 & 0.489 & 2.020 & 7 & 1.959 & 4 & 6439.813 & 0.944 & 1.525 & & 1.594 & \\
\hline 607 & 0.511 & 2.035 & 3 & 1.981 & 6 & 6439.817 & 0.945 & 1.490 & & 1.571 & \\
\hline 73 & 0.305 & 1.786 & 4 & 1.772 & 4 & 6439.819 & 0.947 & 1.448 & & 1.552 & \\
\hline .637 & 0.333 & 1.839 & 1 & 1.801 & 6 & 6439.822 & 0.948 & 1.495 & & 1.576 & \\
\hline 6113.580 & 0.608 & 2.112 & 3 & 2.037 & 4 & 6439.824 & 0.949 & 1.465 & & 1.562 & \\
\hline 6113.616 & 0.623 & 2.117 & 4 & 2.043 & 3 & 6439.827 & 0.950 & 1.451 & & 1.538 & \\
\hline 6113.675 & 0.649 & 2.135 & 4 & 2.061 & 3 & 6441.808 & 0.808 & 2.199 & 7 & 2.124 & 8 \\
\hline 6113.738 & 0.676 & 2.144 & 10 & 2.069 & 7 & 6444.632 & 0.032 & 1.190 & 6 & 1.332 & \\
\hline 6140.601 & 0.314 & 1.778 & 11 & 1.770 & 8 & 6444.711 & 0.066 & 1.263 & 4 & 1.388 & \\
\hline 6140.622 & 0.324 & 1.823 & 6 & 1.796 & 4 & 6444.765 & 0.090 & 1.331 & 7 & 1.433 & \\
\hline 6141.590 & 0.743 & 2.190 & 6 & 2.103 & 3 & 6444.827 & 0.116 & 1.401 & 7 & 1.489 & \\
\hline 6141.651 & 0.769 & 2.220 & 7 & 2.142 & 7 & 4.879 & 0.139 & 1.451 & 4 & 1.515 & 7 \\
\hline 6146.588 & 0.908 & 1.884 & 7 & 1.884 & 7 & 6463.762 & 0.319 & 1.810 & 4 & 1.787 & 4 \\
\hline 6146 & 0.921 & 1.804 & 4 & 1.823 & 10 & 6483.689 & 0.953 & 1.427 & & 1.509 & \\
\hline 634 & 0.928 & 1.747 & 13 & 1.774 & 4 & 6483.691 & 0.954 & 1.395 & & 1.503 & \\
\hline 6148.593 & 0.777 & 2.207 & 10 & 2.131 & 7 & 6483.694 & 0.955 & 1.397 & & 1.483 & \\
\hline 6148.656 & 0.804 & 2.207 & 10 & 2.128 & 10 & 6483.696 & 0.956 & 1.389 & & 1.481 & \\
\hline 6153.584 & 0.939 & 1.600 & 16 & 1.648 & 16 & 6483.699 & 0.957 & 1.381 & & 1.462 & \\
\hline 6153.592 & 0.943 & 1.567 & 21 & 1.627 & 21 & 6483.704 & 0.959 & 1.365 & & 1.457 & \\
\hline 6153.598 & 0.945 & 1.553 & 21 & 1.589 & 21 & 3.707 & 0.961 & 1.334 & & 1.448 & \\
\hline 6153.602 & 0.947 & 1.491 & 21 & 1.572 & 21 & 3.709 & 0.961 & 1.347 & & 1.476 & \\
\hline 3.609 & 0.950 & 1.440 & 31 & 1.549 & 31 & 6483.711 & 0.962 & 1.356 & & 1.485 & \\
\hline 4.597 & 0.710 & 2.207 & 14 & 2.110 & 21 & 6483.714 & 0.964 & 1.321 & & 1.439 & \\
\hline 6164.633 & & 2.191 & 17 & 2.111 & 14 & 3.718 & 0.965 & 1.302 & & 1.435 & \\
\hline 6171 & 0.747 & 2.197 & 8 & 2.109 & 10 & 3.720 & 0.966 & 1.321 & & 1.432 & \\
\hline 917 & 0.398 & 1.967 & 8 & 1.934 & 13 & 6483.723 & 0.967 & 1.307 & & 1.421 & \\
\hline 682 & & 1.574 & 7 & 1.613 & 10 & 3.743 & 0.976 & 1.234 & 16 & 1.372 & 14 \\
\hline 6 & 0.219 & 1.655 & 4 & 1.670 & 10 & 8.636 & 0.096 & 1.344 & 17 & 1.454 & 13 \\
\hline 6412.791 & 0.237 & 1.670 & 6 & 1.680 & 4 & 8.723 & 0.133 & 1.440 & 8 & 1.512 & \\
\hline 6413.846 & 0.694 & 2.170 & 3 & 2.093 & 4 & 6490.639 & 0.964 & 1.317 & 7 & 1.431 & \\
\hline 6413.870 & 0. & 2.187 & 11 & 2.103 & 11 & 6490.695 & 0.988 & 1.194 & 7 & 1.337 & \\
\hline 3.968 & 0.747 & 2.202 & 3 & 2.121 & 3 & 6490.740 & 0.007 & 1.163 & 13 & 1.314 & 11 \\
\hline & & 2.022 & & 1.979 & & 6497.631 & 0.993 & 1.169 & 4 & 1.313 & \\
\hline & 0.894 & 1.990 & & 1.967 & & .668 & 0.009 & 1.174 & 1 & 1.316 & \\
\hline 64 & 0. & 1. & & 1.885 & & 6517.609 & 0.648 & 2.152 & 7 & 2.072 & 8 \\
\hline 6439.706 & 0.898 & 1.953 & & 1.923 & & 6530.682 & 0.312 & 1.803 & 10 & 1.774 & \\
\hline
\end{tabular}

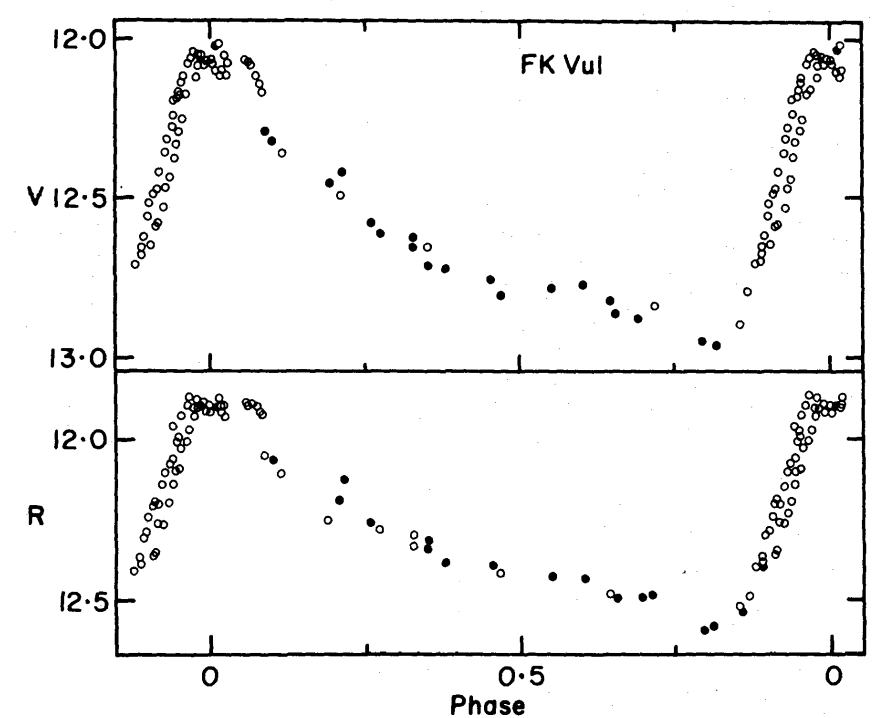

Figure 2. Light curve for FK Vul plotted with a period of 0.4340529 and epoch of maximum of 2446593.88. 


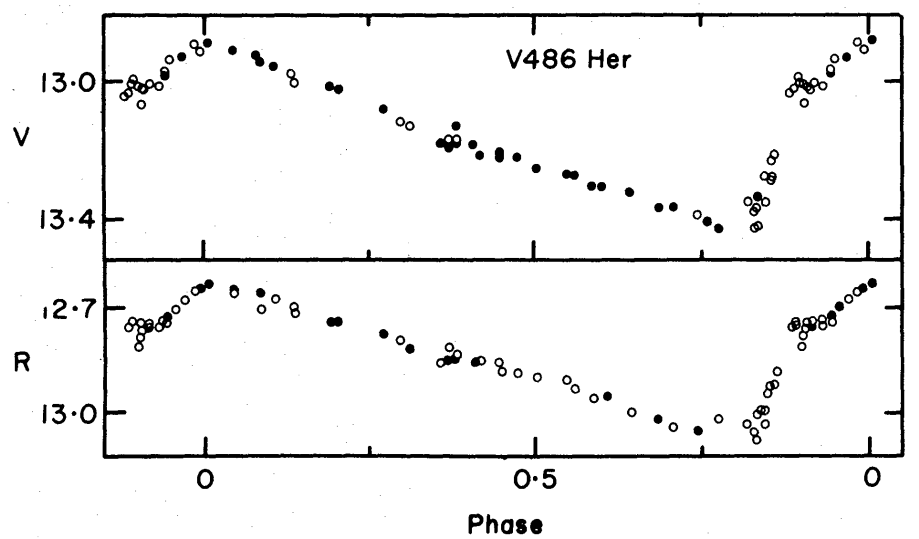

Figure 3. Light curve for V486 Her plotted with a period of 0.8059317 and epoch of maximum of 2446624.83 .

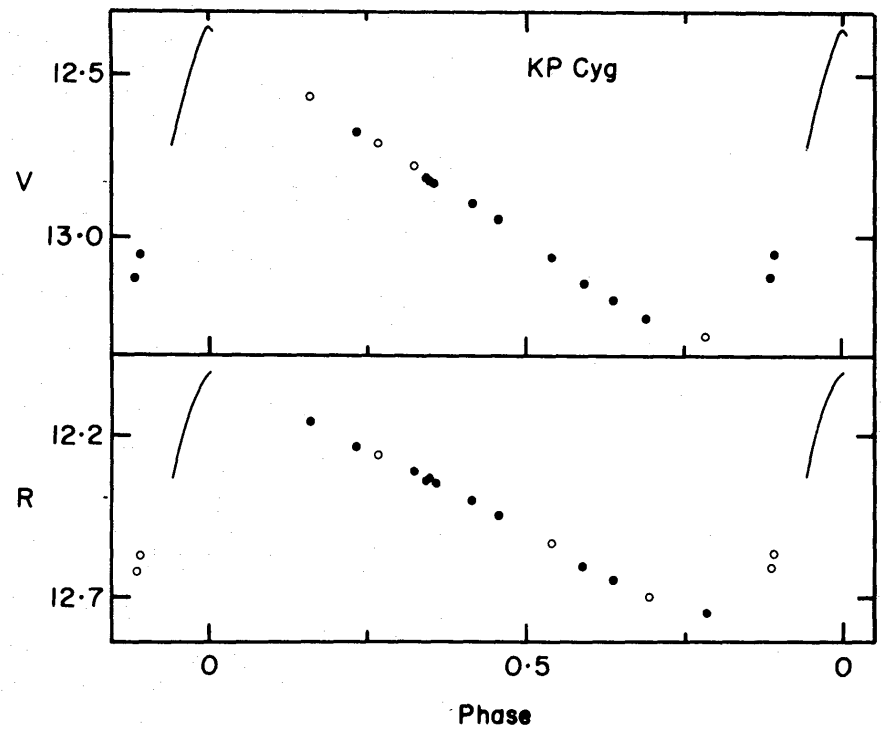

Figure 4. Light curve for KP Cyg plotted with a period of 0.855936 and epoch of maximum of 2446699.62. In this figure and subsequent figures, the points during rising light are very numerous and too crowded to be plotted individually. The solid curve shows their locus.

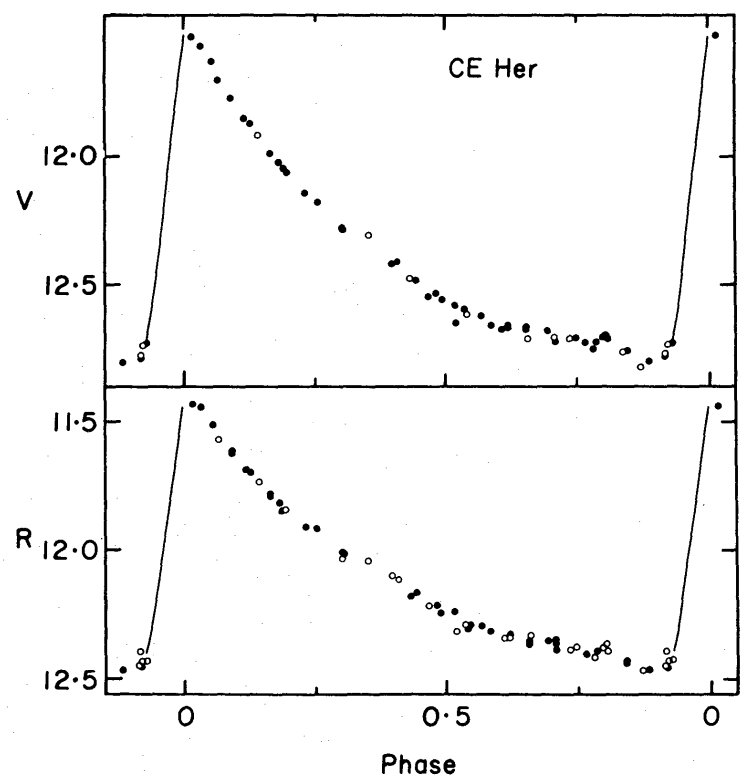

Figure 5. Light curve for CE Her plotted with a period of 1.2094357 and epoch of maximum of 2446583.77 . 


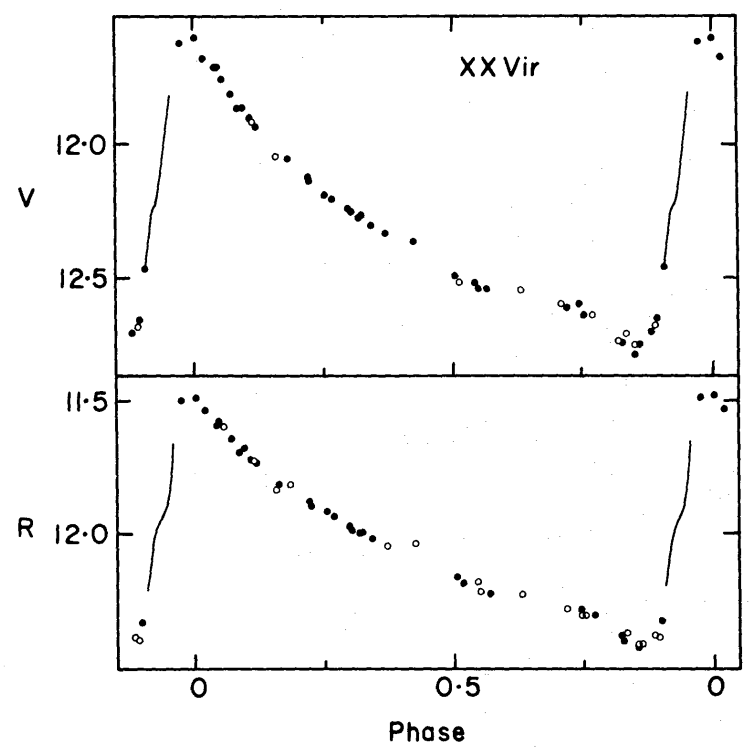

Figure 6. Light curve for XX Vir plotted with a period of 1.3482051 and epoch of maximum of 2446203.67.

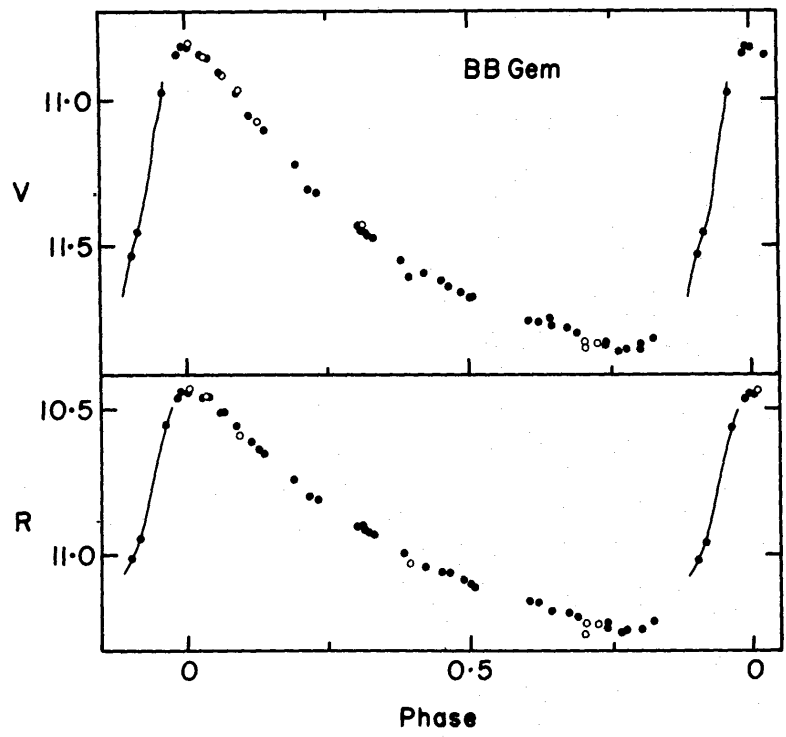

Figure 7. Light curve for BB Gem plotted with a period of 2.308207 and epoch of maximum of 2446056.78 .

the exception of KP Cyg). Differential measurements were made between the check stars and the comparison stars at random times. While it is common practice to observe the check stars every night, the present procedure is less time consuming and serves to alert us to any potential variability in our comparison stars. Additionally, the constancy of the magnitudes of the comparison stars on photometric nights was found to be consistent with the usual photometric errors. There is thus little likelihood that any variation as great as $0.01 \mathrm{mag}$ would have escaped our notice.

In Table 9 we list the comparison and check stars for each variable. They are identified by the variable name in column one. Co indicates a comparison star and Ch a check star. Columns 2 and 3 give the coordinates of the check and comparison stars. The mean magnitudes, colours and their standard errors, calculated from the nights which were judged to be photometric, are listed in columns 4-9. The last column gives the number of nights included in the means. 
Table 9. Comparison and check stars.

\begin{tabular}{|c|c|c|c|c|c|c|c|c|c|}
\hline Star* & RA (1S & 50) Dec & $\mathrm{V}$ & se & $\mathrm{R}$ & se & $V-R$ & se & $\mathrm{n}$ \\
\hline CM UMa Co & $9: 40: 16$ & $49: 44: 59$ & 10.667 & 7 & 10.310 & 8 & 0.358 & 3 & 5 \\
\hline CM UMa Ch & $9: 40: 08$ & $49: 44: 57$ & 10.438 & 8 & 10.153 & 10 & 0.285 & 4 & 3 \\
\hline FK Vul Co & $20: 50: 18$ & $22: 26: 20$ & 10.245 & 6 & 9.916 & 7 & 0.329 & 2 & 7 \\
\hline $\mathrm{FK}$ Vul $\mathrm{Ch}$ & $20: 50: 27$ & $22: 23: 57$ & 11.202 & 7 & 10.883 & 9 & 0.319 & 4 & 6 \\
\hline V486 Her Co & $17: 24: 32$ & $27: 15: 31$ & 7.591 & 5 & 7.530 & 5 & 0.158 & 3 & 9 \\
\hline V486 Her Ch & $17: 24: 59$ & $27: 14: 37$ & 10.748 & 6 & 10.233 & 6 & 0.515 & 5 & 5 \\
\hline KP Cyg Co & $20: 04: 49$ & $41: 02: 39$ & 9.356 & 10 & 8.833 & 9 & 0.522 & 6 & 5 \\
\hline CE Her Co & $17: 37: 47$ & $15: 19: 38$ & 10.997 & 5 & 10.812 & 6 & 0.186 & 2 & 11 \\
\hline $\mathrm{CE}$ Her $\mathrm{Ch}$ & $17: 40: 00$ & $15: 09: 44$ & 11.312 & 5 & 10.987 & 6 & 0.325 & 3 & r \\
\hline $\mathrm{XX}$ Vir Co & $14: 13: 06$ & $-5: 53: 06$ & 9.870 & 9 & 9.710 & 9 & 0.152 & 2 & 9 \\
\hline $\mathrm{XX}$ Vir $\mathrm{Ch}$ & $14: 13: 04$ & $-6: 10: 20$ & 10.143 & 10 & 9.747 & 10 & 0.396 & 3 & 4 \\
\hline $\mathrm{BB}$ Gem Co & $6: 31: 39$ & $13: 03: 42$ & 9.648 & 5 & 9.128 & 5 & 0.521 & 1 & 6 \\
\hline BB Gem Ch & $6: 31: 43$ & $13: 04: 52$ & 10.321 & 6 & 10.102 & 6 & 0.219 & 3 & 6 \\
\hline
\end{tabular}

Owing to difficulties in determining the period of CMUMa as discussed in the next section, further observations of that star were undertaken on six nights during 1988 January and February with the newly commissioned CCD camera at Behlen Observatory. This instrument and data reduction procedures are described by Schmidt (1988). The instrument was new at that time and its performance was not fully optimized. Consequently, the data quality is not completely consistent among the various nights used and the photometry was not reduced accurately to the standard system. For these reasons, we do not tabulate the data here. However, they are fully adequate for the purpose of resolving the period of CMUMa and it will be seen that the light curve from the CCD is in very good agreement with that from the photoelectric data.

\section{Discussion of individual stars}

\subsection{UMa}

When it became obvious that the period listed in the GCVS for this star (1.44 day) was grossly in error, we tried to employ the phase dispersion minimization technique (Stellingwerf 1978) to search for a new period in our data. However, we could not definitively establish the period owing to the relatively long intervals between the observations which made the cycle count ambiguous.

When the star again became available in 1988 January we obtained further data with the CCD camera. An effort was made to obtain data on successive nights and over as long an interval on a given night as possible. Two consecutive nights with 6 and $9 \mathrm{hr}$ of coverage eliminated any period shorter than half a day. The fit of all the CCD data then yielded a period of 0.589 days. It should be noted that the cycle count between successive years of data is still ambiguous so we can not refine this period by combining all the data. We give a date of maximum for each year in Table 1.

The light curves for CM UMa are plotted in Fig. 1. The upper panels contain the $V$ and $R$ data from the photoelectric photometer (which is listed in Table 2) while the CCD data are plotted in the lower panel. It can be seen that the light curve is typical for a type ab RR Lyraestar. A comparison of the photoelectric and the CCD light curves in Fig. 1 shows excellent agreement in the overall shape and the actual magnitude levels. The scatter during rising light and after maximum is likely to be due to the Blazhko effect.

Owing to the short time over which the CCD observations were obtained (31 days) compared to the interval for the photoelectric observations (438 days), the Blazhko effect is less evident in the former. However, there is excess scatter in the light curve around phase 0.4 . Since the 
CCD camera was new, we might wonder whether this is an instrumental effect or is due to the star itself. We prefer the latter interpretation for several reasons. In the first place, this region of the light curve was observed with the CCD on three different nights. In each case the data agree well before and after this region but the star follows a different curve (but a curve with little scatter) through this region on each of the three nights. Secondly, photometry for other stars of comparable brightness observed with the CCD on the same nights shows scatter much too small to explain the scatter in this part of the light curve of CMUMa. Finally, other RR Lyrae stars of similar period show similar scatter in their light curves (see, e.g. Lub 1977).

\subsection{FK Vul}

This star was included in our programme because the period given in the third edition of the GCVS, 0.77 day, fell within the range of interest. The fourth edition has corrected this period to 0.4340529 day. From the phase dispersion minimization analysis of our data we obtained a period of 0.43404 day, in good agreement. Subjecting the observations to Fourier decomposition yielded the coefficients listed in Table 10. The quantities are as defined by Simon \& Lee (1981). The amplitude ratio, $R(21)$, and phase differences, $\phi(21)$ and $\phi(31)$, for FK Vul place it among the RRab stars (Simon \& Teays 1982), a classification which is also consistent with its period and amplitude. The scatter (in excess of observational error) apparent in Fig. 2 is likely due to the Blazhko effect which is common in RR Lyrae stars with periods like that of FK Vul.

Table 10. Fourier coefficients of the light curves.

\begin{tabular}{|c|c|c|c|c|c|c|c|c|}
\hline Star & $\begin{array}{l}\text { Drder } \\
\text { pf fit }\end{array}$ & $\begin{array}{l}\text { Std. Dev. } \\
\text { (mag) }\end{array}$ & $\stackrel{\text { Ampl }}{(\mathrm{mag})}$ & $R(21)$ & $\phi(21)$ & $R(31)$ & $\phi(31)$ & $\phi(41)$ \\
\hline FK VUI & 5 & 0.056 & 0.975 & 0.565 & 3.92 & 0.299 & 2.01 & 6.09 \\
\hline V486 Her & 5 & 0.035 & 0.551 & 0.459 & 4.54 & 0.206 & 3.11 & $\ldots$ \\
\hline KP Cyg & 5 & 0.022 & 0.966 & 0.442 & 4.52 & 0.224 & 2.47 & 6.77 \\
\hline CE Her & 8 & 0.037 & 1.36 & 0.480 & 4.06 & 0.375 & 1.96 & 6.20 \\
\hline $\mathrm{xX}$ vir & 8 & 0.037 & 1.19 & 0.535 & 4.21 & 0.369 & 2.22 & 6.73 \\
\hline BB Gem & 7 & 0.262 & 1.06 & 0.471 & 4.08 & 0.240 & 2.10 & 6.11 \\
\hline
\end{tabular}

\section{$3.3 \mathrm{~V} 486 \mathrm{Her}$}

The light curve for this star is plotted in Fig. 3. The long period (compared with most RR Lyrae stars) and low amplitude of this star make it comparable to XZ Cet (Simon \& Teays 1982) which is best modelled as an anomalous Cepheid (Teays \& Simon 1985) similar to the cluster variable NGC5466-V19 (Zinn \& Dahn 1976). Indeed, the values of $\phi(21)$ and $\phi(31)$ displayed in Table 10 place V486 Her between the RRab stars and XZ Cet on the corresponding Fourier diagrams. However, the amplitude ratio $R(21)$ for $\mathrm{V} 486 \mathrm{Her}$ agrees with the RR Lyr stars. This is in sharp contrast to both XZ Cet and NGC 5466-V19 whose small values of $R(21)$ set them apart from the RRab stars of similar period. Although this may indicate that V486Her is an RRabstar, the unusual still-stand and sudden change in slope about threequarters of the way up the rising branch of the light curve raise doubts. The star needs further study. 


\subsection{KP Cyg}

The light curve for this star is presented in Fig. 4. While the phase coverage is not fully adequate for Fourier decomposition, the lower order coefficients seem stable enough for inclusion in Table 10. The coefficients are consistent with the Fourier diagrams for both field RR Lyr stars (Simon \& . Teays 1982) and type II Cepheids (Simon 1986).

A solar metallicity $(\Delta S=0)$ was found by Preston (1959) for KP Cyg. This, too, is consistent with either type. We are therefore unable to assign this star to a class on the basis of present information.

If KP Cyg turns out to be a type II Cepheid, it will have the shortest period among this class. This would extend the domain of these objects well below 1 day. For this reason, further study of KP Cyg and similar stars is needed.

\subsection{CE Her AND XX Vir}

Our light curves for these stars are plotted in Figs 5 and 6. Both of these stars were assigned by Diethelm (1983) to the class he designated RRd. A light curve for XX Vir was analysed by Simon (1986) who classified it as a type II Cepheid of subclass S-XX. The Fourier parameters from our light curve (Table 10) agree with his. Those for CE Her also place it in the S-XX subclass.

In both CE Her and XX Vir there is excessive scatter before minimum. Fig. 8 shows the last part of declining light for both stars on an expanded scale with error bars. The observations were made during a number of cycles as indicated by different symbols. At phases $0.52-0.55$ and 0.66 in CE Her and phases 0.54 and 0.82 in XX Vir, the scatter between different cycles is large compared with the error bars. We note that at this magnitude level and fainter our internal errors for other stars realistically represent the scatter in the light curves.

There are also bumps and dips in the light curves which seem to repeat from cycle to cycle. For example, there is a local maximum at phase 0.8 in $\mathrm{CEHer}$ which is established by three

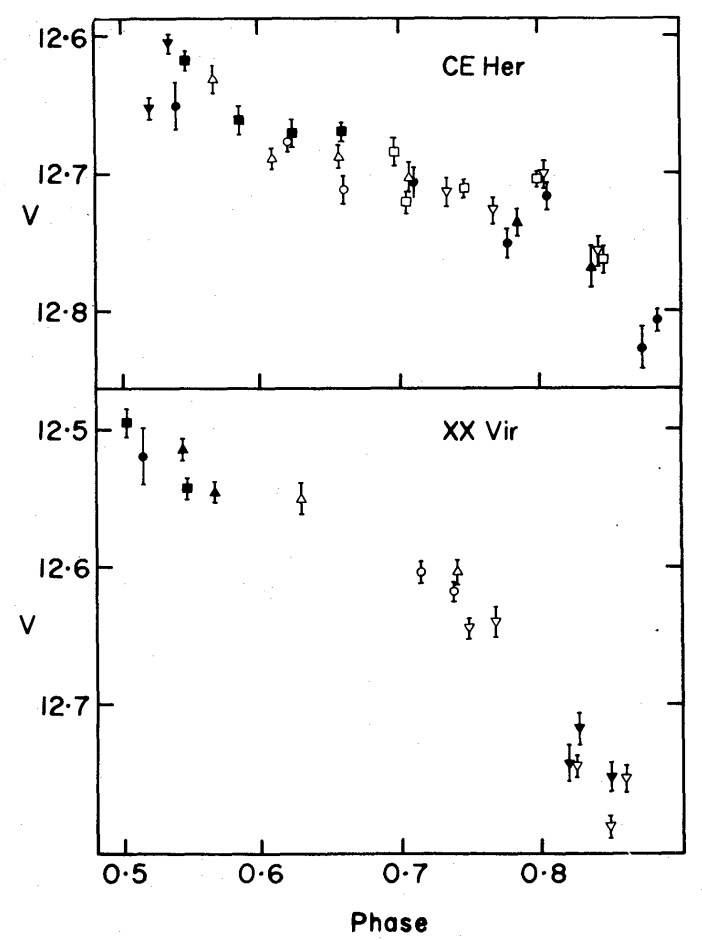

Figure 8. Expanded plots of the last part of declining light in CE Her and XX Vir. When only one point is present for a particular night, it is plotted as a filled circle. Otherwise, each night is represented by a different symbol. 
concordant data points which are also well determined. The dip near phase 0.6 is also significant. Looking at the expanded light curve for XX Vir we see that the behaviour is not so well delineated but is consistent with what we see in CE Her.

- This behaviour is complex and can be interpreted in various ways. It is possible to draw several straight lines through the variation of $\mathrm{CE} H e r$ with breaks near phases $0.54,0.63$ and 0.79 . On the other hand, we might consider the bumps, dips and intervals of excessive scatter to be small, relatively rapid fluctuations about a smooth overall decline. In any event, we suggest that this behaviour is indicative of the pulsational properties of these stars and should be pursued further.

\subsection{BB Gem}

Based on its location, Diethelm (1983) considers this star to be a classical Cepheid. Fourier coefficients derived from our light curve (Fig. 7) are listed in Table 10. Plotting these in the Fourier diagrams for both the type II (Simon 1986) and classical (Simon \& Moffett 1985) Cepheids we find the phases $\phi(21), \phi(31)$ and $\phi(41)$ to be discrepant with type II stars of similar period such as UX Nor and V465 Oph. On the other hand, the Fourier parameters for BB Gem fit extremely well with the sequence of classical Cepheids. Thus, the Fourier decomposition strongly supports Diethelm's classification. Since the well studied classical Cepheid SU Cas ( $P=1.95$ day) is almost certainly an overtone pulsator (Gieren 1982; Aikawa, Antonello \& Simon 1987), BB Gem becomes the shortest period fundamental mode pulsator known among the classical Cepheids in the Galaxy.

\section{Acknowledgements}

Three of the stars were taken from a list of pulsating variables with periods near 0.8 day compiled by T. J. Teays. This proved very useful in setting up our program and we are grateful to him for providing it. The phase dispersion minimization program used in this project was kindly supplied by R. F. Stellingwerf.

\section{References}

Aikawa, T., Antonello, E. \& Simon, N. R. 1987. Astr. Astrophys., 181, 25.

Diethelm, R., 1983. Astr. Astrophys., 124, 108.

Diethelm, R., 1986. Astr. Astrophys. Suppl., 64, 261.

Gieren, W., 1982. Publs astr. Soc. Pacif., 94, 360.

Harris, H. C., 1981. Astr. J., 86, 719.

Harris, H. C. \& Wallerstein, G., 1984. Astr. J., 89, 379.

Landolt, A. U., 1983. Astr. J., 88, 439.

Lub, J., 1977. Astr. Astrophys. Suppl., 29, 345.

Peterson, J. O. \& Diethelm, R., 1986, Astr. Astrophys., 156, 337.

Preston, G. W., 1959. Astrophys. J., 130, 507.

Schmidt, E. G., 1988. Proc. Ninth Annual Fairborn-Smithsonian-IAPPP Symposium, ed. Hayes, D., Fairborn Observatory Press.

Simon, N. R., 1986. Astrophys. J., 311, 305.

Simon, N. R. \& Lee, A. S., 1981. Astrophys. J., 248, 291.

Simon, N. R. \& Moffett, T. J., 1985. Publs. Astr. Soc. Pacif., 97, 1078.

Simon, N. R. \& Teays, T. J., 1982. Astrophys. J., 261, 586.

Stellingwerf, R. F., 1978. Astrophys. J., 224, 953.

Taylor, D. J., 1980. Publs astr. Soc. Pacif., 92, 108.

Teays, T. J. \& Simon, N. R., 1985. Astrophys. J., 290, 683.

Wallerstein, G. \& Cox, A. N., 1984. Publs astr. Soc. Pacif., 96, 677.

Zinn, R. \& Dahn, C. C., 1976. Astr. J., 81, 527. 